\title{
Exterior Trajectory Modelling Method Selection and Analysis for Simulation Test of Guided Bomb
}

\author{
Yuan Gao ${ }^{1,2}$ a , Hongyu Zhai ${ }^{1, b}$, Hongquan $\mathrm{Wu}^{2}$, Guangling Dong ${ }^{2}$, Chi He $\mathrm{He}^{2, \mathrm{c}}$ \\ ${ }^{1}$ College of Computer, Changchun University of Science and Technology, 7089 Weixing Road, \\ Changchun, 130022, China \\ Baicheng Ordnance Test Center of China, P.O.B 108, Baicheng, 137001, China \\ aemail: wutong413@163.com, bemail:7604888@qq.com, cemail: hechi119@sina.com
}

Keywords: Body Coordinate System; Trajectory Model; Mathematical Simulation

\begin{abstract}
Two exterior trajectory models of guided bomb in aircraft coordinate system (ACS) and body coordinate system (BCS) are built for simulation and analysis under different meteorological conditions. Simulation errors of different modelling methods are studied and analyzed to verify the rationality of modelling method adopted in this paper, which also indicates the efficient path to decrease simulation error and improve precision.
\end{abstract}

\section{Introduction}

Simulation test technology provides strong support for index examination, boundary condition simulation, test scheme optimization, failure recurrence, etc. [1] for type approval test and evaluation in proving ground, where, suitable modeling method is an efficient path to improve simulation precision. As to fin stabilized guided bomb, there should be minor difference among different modeling methods of a precise simulation model theoretically. However, owing to different application scopes of each modeling method, simulation test results with various exterior trajectory models could take on different modelling error. In order to decrease modeling error, we build an exterior trajectory model for winged low speed spinning bomb based on specific aerodynamic model that can be used for simulation in different meteorological conditions and certain application scopes. Besides, simulation results of uncontrolled free flight trajectory with different modelling methods are compared, which are used to analyze their feasibility and applicability.

\section{Modeling Method of Uncontrolled Free Flight Trajectory}

Research on trajectory models abroad starts earlier, and has put forward many modeling methods as Yourdon method [2], Booch method [3], OMT method, etc. which take on various scopes of application. In principle, frequently-used exterior trajectory modeling methods in simulation mainly include two types as body coordinate system (BCS) based method [4] and aircraft coordinate system (ACS) based method. The former is suitable for exterior trajectory modeling of uncontrolled high-speed rotating projectile with axial or rotational symmetry aerodynamic configurations. This type of projectiles take on major maneuver on pitching direction of flight trajectory, while their azimuth maneuver are relatively minor compared to controlled projectile, which is mainly caused by factors as wind, gyro moment from self-high-speed rotation, etc. Coordinate systems used generally include: earth coordinate system, translational coordinate system, ballistic coordinate system, velocity coordinate system, first projectile axis coordinate system, second projectile axis coordinate system [5] and BCS, etc. Transformation of each coordinate system is as follows: turn the first angel around positive z-axis, then turn the second angel around negative $y$-axis of intermediate coordinate system (if the third turning is needed, making rotation around positive $\mathrm{x}$-axis of the second intermediate coordinate system). Namely, vertical turning is taken before lateral turning. Aerodynamic forces are generally given out from the second projectile axis coordinate system to velocity coordinate system. Dynamics and kinematic equations of mass center 
are generally given out in earth coordinate system or velocity coordinate system. Primary attitude parameters include swinging angles in vertical and lateral axes of projectile, attack angles in vertical and lateral direction, etc. Velocity coordinate system is generally defined as a non-roll coordinate system, where attack angle is given out relative to the second projectile axis coordinate system, and swinging angle relative to the first projectile axis coordinate system.

ACS-based exterior trajectory modeling method is mainly coping with controlled projectiles, which take on major maneuver in azimuth direction. Coordinate systems used mainly include: earth coordinate system, translational coordinate system, ballistic coordinate system (non-rolling velocity coordinate system), velocity coordinate system, BCS, etc. Transformation of each coordinate system is as follows: turn the first angel around positive y-axis, then turn the second angel around negative z-axis of intermediate coordinate system (if the third turning is needed, making rotation around positive $\mathrm{x}$-axis of the second intermediate coordinate system). Namely, lateral turning is taken before vertical turning. Attitude angles of airframe mainly include: pitching angle, yawing angle, attack angle and slipping angle, etc. Besides, dynamic model in non-rotating coordinate system also takes on velocity rolling angle [6]. Kinematic and dynamical equations are generally based on earth coordinate system, velocity coordinate system, non-rolling velocity coordinate system, etc. which takes on different objects and scopes.

Aerodynamic forces on controlled rolling projectiles are generally given out in quasi-velocity coordinate system (ballistic coordinate system), while aerodynamic forces of non-rolling projectiles are given out in velocity coordinate system. As to different models with same modeling method, their dynamics and kinematic equation around mass center are built in BCS, whose chief difference lie in their kinematic equation of mass center. In this paper, we penetrate into modelling methods of typical winged low speed spinning guided bomb with axial symmetry aerodynamic configurations, which is changeable in flight process and takes on major maneuver in both pitching and azimuth directions. So, the second type of method is suitable for exterior trajectory modeling, which uses $\mathrm{BCS}$ for kinematics and dynamic modelling of mass center.

\section{Exterior Trajectory Models}

Adopting aircraft coordinate system based modeling method for building trajectory model of non-rolling guided bomb, assumptions as follows need to be considered: aerodynamic configuration of projectile is ideal axial symmetry without mass eccentric and configurational asymmetry; meteorological conditions are standard and windiness; earth surface is plane, gravity acceleration is constant and perpendicular down to earth surface; taking no account of earth rotation and Coriolis inertia force, earth coordinate system as an inertial coordinate system [7]. In modeling process, earth coordinate system $A x y z$, ballistic coordinate system $O x_{2} y_{2} z_{2}$, velocity coordinate system $O x_{3} y_{3} z_{3}$ and BCS $O x_{1} y_{1} z_{1}$ are taken as reference frames.

Dynamical equations of projectile mass center are built on BCS $O x_{1} y_{1} z_{1}$, with earth coordinate system $A x y z$ as static coordinate system and BCS $O x_{1} y_{1} z_{1}$ as dynamic coordinate system.

$$
\frac{d \vec{V}}{d t}=\frac{\delta \vec{V}}{d t}+\vec{\omega} \times \vec{V}=\sum \vec{F}_{v} / m
$$

Where, $\vec{V}$ is velocity relative to static coordinate system (ECS), $\mathrm{m} / \mathrm{s} ; d \vec{V} / d t$ is acceleration relative to static coordinate system (ECS), $\mathrm{m} / \mathrm{s} 2 ; d \vec{V} / d t-\vec{V}$ is phasor difference speed relative to dynamic coordinate system $(\mathrm{BCS}), \mathrm{m} / \mathrm{s} ; \vec{\omega}$ is rotation angular velocity of BCS to ECS, $\mathrm{rad} / \mathrm{s}$.

Components of $\vec{\omega}$ in each axis of BCS are $\omega x_{1}, \omega y_{1}, \omega z_{1}$; components of $\vec{V}$ in each axis of BCS are $V_{x 1}, V_{y 1}, V_{z 1}$; components of missile resultant force on each axis of BCS are $F_{x 1}, F_{y 1}, F_{z 1}$.

$$
\vec{\Omega} \times \vec{V}=\left[\begin{array}{ccc}
\vec{x}_{1} & \vec{y}_{1} & \vec{z}_{1} \\
\omega_{x 1} & \omega_{y 1} & \omega_{z 1} \\
V_{x 1} & V_{y 1} & V_{z 1}
\end{array}\right]=\left[\begin{array}{c}
\omega_{y 1} V_{z 1}-\omega_{z 1} V_{y 1} \\
\omega_{z 1} V_{x 1}-\omega_{x 1} V_{z 1} \\
\omega_{x 1} V_{y 1}-\omega_{y 1} V_{x 1}
\end{array}\right]
$$


Projections of $\frac{\delta \vec{V}}{d t}$ on each axis of BCS are

$\left(\frac{\delta \vec{V}}{d t}\right)=\left[\begin{array}{lll}d V_{x 1} / d t & d V_{y 1} / d t & d V_{z 1} / d t\end{array}\right]^{\mathrm{T}}$

Dynamical equation of mass center on BCS is

$\left[\begin{array}{l}d V_{x 1} / d t \\ d V_{y 1} / d t \\ d V_{z 1} / d t\end{array}\right]+\left[\begin{array}{c}\omega_{y 1} V_{z 1}-\omega_{z 1} V_{y 1} \\ \omega_{z 1} V_{x 1}-\omega_{x 1} V_{z 1} \\ \omega_{x 1} V_{y 1}-\omega_{y 1} V_{x 1}\end{array}\right]=\left[\begin{array}{c}F_{x 1} \\ F_{y 1} \\ F_{z 1}\end{array}\right] / m$

Kinematic equation around mass center is the same as general bomb, which is given directly as follows

$$
\begin{gathered}
{\left[\begin{array}{c}
J_{x 1} \frac{d \omega_{x 1}}{d t} \\
J_{y 1} \frac{d \omega_{y 1}}{d t} \\
J_{z 1} \frac{d \omega_{z 1}}{d t}
\end{array}\right]+\left[\begin{array}{c}
\omega_{y 1} \omega_{z 1}\left(J_{z 1}-J_{y 1}\right) \\
\omega_{z 1} \omega_{x 1}\left(J_{x 1}-V_{z 1}\right) \\
\omega_{x 1} \omega_{y 1}\left(J_{y 1}-J_{x 1}\right)
\end{array}\right]=\left[\begin{array}{c}
M_{x 1} \\
M_{y 1} \\
M_{z 1}
\end{array}\right]} \\
{\left[\begin{array}{c}
\dot{\gamma} \\
\dot{\psi} \\
\dot{\varphi}
\end{array}\right]=\left[\begin{array}{c}
\omega_{x 1}-\tan \varphi\left(\omega_{y 1} \cos \gamma-\omega_{z 1} \sin \gamma\right) \\
\frac{1}{\cos \varphi}\left(\omega_{y 1} \cos \gamma-\omega_{z 1} \sin \gamma\right) \\
\omega_{y 1} \sin \gamma+\omega_{z 1} \cos \gamma
\end{array}\right]}
\end{gathered}
$$

Where, $M_{x 1}, M_{y 1}$ and $M_{z 1}$ are moment components of all external forces on missile mass center in each axis of BCS. $J_{x 1}, J_{\mathrm{y} 1}$ and $J_{z 1}$ are rotational inertia of projectile to each axis of BCS; $\omega_{x 1}, \omega_{y 1}$, and $\omega_{z 1}$ are components of $\vec{\omega}$ in each axis of $\operatorname{BCS} ; \varphi$ is pitching angle; $\psi$ is azimuth angel; $\gamma$ is rolling angel.

Components of missile velocity in ECS are

$$
\left[\begin{array}{lll}
V_{x} & V_{y} & V_{z}
\end{array}\right]^{\mathrm{T}}=\left[\begin{array}{lll}
\frac{d x}{d t} & \frac{d y}{d t} & \frac{d z}{d t}
\end{array}\right]^{\mathrm{T}}
$$

According to definition of ballistic coordinate system $O x_{2} y_{2} z_{2}$, velocity vector $V$ coincides with $O x_{2}$ axis. With transformation matrix between ballistic coordinate system and earth coordinate system, we get

$$
\left[\begin{array}{l}
V_{x} \\
V_{y} \\
V_{z}
\end{array}\right]=L^{T}\left(\psi_{V}, \theta\right)\left[\begin{array}{l}
V_{x 2} \\
V_{y 2} \\
V_{z 2}
\end{array}\right]=L^{T}\left(\psi_{V}, \theta\right)\left[\begin{array}{l}
V \\
0 \\
0
\end{array}\right]=\left[\begin{array}{c}
V \cos \theta \cos \psi_{V} \\
V \sin \theta \\
-V \cos \theta \sin \psi_{V}
\end{array}\right]
$$

Then, kinematic equation of mass center is

$$
\left[\begin{array}{lll}
\frac{d x}{d t} & \frac{d y}{d t} & \frac{d z}{d t}
\end{array}\right]^{\mathrm{T}}=\left[\begin{array}{llll}
V \cos \theta \cos \psi_{V} & V \sin \theta & -V \cos \theta \sin \psi_{V}
\end{array}\right]^{\mathrm{T}}
$$

Using transformation matrix between earth coordinate system and BCS, we get

$V_{x}=\cos \phi \cos \psi V_{x 1}+(-\sin \phi \cos \psi \cos \gamma+$

$$
\sin \psi \sin \gamma) V_{y 1}+(\sin \phi \cos \psi \sin \gamma+\sin \psi \cos \gamma) V_{z 1}
$$

$V_{y}=\sin \varphi V_{x 1}+\cos \varphi \cos \gamma V_{y 1}-\cos \varphi \sin \gamma V_{z 1}$

$V_{z}=(\sin \phi \sin \psi \cos \gamma+\cos \psi \sin \gamma) V_{z 1}-\cos \phi \sin \psi V_{x 1}$

$+(-\sin \phi \sin \psi \sin \gamma+\cos \psi \cos \gamma) V_{z 1}$

Angular relations in exterior trajectory model are as follows

$\sin \beta=\cos \theta\left(\cos \gamma \sin \left(\psi-\psi_{v}\right)+\sin \varphi \sin \gamma \cos \left(\psi-\psi_{v}\right)\right)-\cos \varphi \sin \gamma \sin \theta$ 
$\sin a=\left(\cos \theta\left(\sin \varphi \cos \gamma \cos \left(\psi-\psi_{v}\right)-\sin \gamma \sin \left(\psi-\psi_{v}\right)\right)-\sin \theta \cos \varphi \cos \gamma\right) / \cos \beta$

$\sin \gamma_{v}=(\cos a \sin \beta \sin \varphi-\sin a \sin \beta \cos \gamma \cos \varphi+\cos \beta \sin \gamma \cos \varphi) / \cos \theta$

Where, $\beta$ is sideslip angle; $\psi_{v}$ is trajectory deflection angle; $\gamma_{v}$ is velocity rolling angel; $\alpha$ is attack angel; $\theta$ is trajectory inclination angle; $\varphi$ is pitching angel; $\psi$ is azimuth angel; $\gamma$ is rolling angel.

\section{Exterior Trajectory Model in Windy Condition}

Under windy conditions, relative trajectory coordinate system $O x_{4} y_{4} z_{4}$ is introduced, whose mutual directions can be determined by two angels of $\psi_{v r}$ and $\theta_{r}$. The computing formula of relative inclination and deflection angels are as follows:

$$
\begin{aligned}
& {\left[\begin{array}{c}
\cos \theta_{r} \cos \psi_{v r} \\
\sin \theta_{r} \\
-\cos \theta_{r} \sin \psi_{v r}
\end{array}\right]=\left[\begin{array}{c}
v_{x}-w_{x} \\
v_{y} \\
v_{z}-w_{z}
\end{array}\right] 1 / v_{r}} \\
& v_{v}=\sqrt{\left(v_{x}-w_{x}\right)^{2}+\left(v_{y}-w_{y}\right)^{2}+\left(v_{z}-w_{z}\right)^{2}} \\
& \theta_{r}=\arcsin \left(v_{y} / v_{r}\right) \\
& \psi_{v r}=-\arcsin \left(\left(v_{z}-w_{z}\right) / v_{r} \cos \theta_{r}\right)
\end{aligned}
$$

Correspondingly, geometrical relation equations of non-rolling missile are

$$
\begin{aligned}
\sin \beta_{r}= & \cos \theta_{r}\left(\cos \gamma \sin \left(\psi-\psi_{v r}\right)+\sin \varphi \sin \gamma \cos \left(\psi-\psi_{v r}\right)\right)-\cos \varphi \sin \gamma \sin \theta_{r} \\
\sin \alpha_{r}= & \left(\cos \theta_{r}\left(\sin \varphi \cos \gamma \cos \left(\psi-\psi_{v r}\right)-\sin \gamma \sin \left(\psi-\psi_{v r}\right)\right)\right. \\
& \left.-\sin \theta_{r} \cos \varphi \cos \gamma\right) / \cos \beta_{r} \\
\cos \gamma_{v r} & =\left(\cos \gamma \cos \left(\psi-\psi_{v r}\right)-\sin \varphi \sin \gamma \sin \left(\psi-\psi_{v r}\right)\right) / \cos \beta_{r}
\end{aligned}
$$

Where, $\beta_{r}$ is relative sideslip angle; $\alpha_{r}$ is relative attack angel; $\theta_{r}$ is relative trajectory inclination angle; $\psi_{v r}$ is relative trajectory deflection angel; $\gamma_{v r}$ is relative velocity rolling angel; $\varphi$ is pitching angel; $\psi$ is azimuth angel; $\gamma$ is rolling angel.

\section{Computational Model of Aerodynamic Force and Moment}

Calculation of aerodynamic force and moment depends on aerodynamic force data, which is often obtained from wind-tunnel blowing test in certain significant conditions. Whereas aerodynamic coefficients besides blowing test points are obtained with interpolation method in simulation. This method is complicated and trivial with big interpolation error, which is caused by many interpolation dimensions and multiple variable relationships of aerodynamic force and moment coefficients. As fitting method herein takes on relatively minor error, aerodynamic coefficients besides blowing test points can be calculated via fitting equation (1), which not only guarantees computational accuracy but also simplifies computational complexity.

$$
\left.\begin{array}{l}
C_{x}, C_{y}, C_{z}, \\
m_{x 1}, m_{y 1}, m_{z 1} \\
M_{x z 1}, M_{z y 1}, M_{z z 1}
\end{array}\right\}=f\left(M, \alpha, \beta, \delta, \omega_{x 1}, \omega_{y 1}, \omega_{z 1}\right)
$$

The above equation forms the computational model of aerodynamic force and moment.

Aerodynamic resistance on projectile body is $R_{x}=C_{x} q S=0.5 \rho S C_{x} V^{2}, c_{x}=c_{0}+c_{x_{a^{2}}}\left(\delta^{2}\right)$.

Projection of resistance in BCS is

$$
\left[\begin{array}{l}
R_{x x 1} \\
R_{x y 1} \\
R_{x z 1}
\end{array}\right]=L(\beta, \alpha)\left[\begin{array}{c}
R_{x} \\
0 \\
0
\end{array}\right]=\left[\begin{array}{ccc}
\cos \alpha \cos \beta & \sin \alpha & -\cos \alpha \sin \beta \\
-\sin \alpha \cos \beta & \cos \alpha & \sin \alpha \sin \beta \\
\sin \beta & 0 & \cos \beta
\end{array}\right]\left[\begin{array}{c}
R_{x} \\
0 \\
0
\end{array}\right]=\left[\begin{array}{c}
\cos a \cos \beta \\
-\sin a \cos \beta \\
\sin \beta
\end{array}\right] R_{x}
$$


Projectile lift force is $R_{y}=0.5 \rho S C_{y} V^{2}, \quad C_{y}=c_{y}^{\prime} a, \quad c_{y}^{\prime}=c_{y_{a}}+c_{y_{a^{3}}} a^{2}$. Projection of Lift force in BCS is

$$
\left[\begin{array}{l}
R_{y x 1} \\
R_{y y 1} \\
R_{y z 1}
\end{array}\right]=L(\beta, \alpha)\left[\begin{array}{c}
0 \\
R_{y} \\
0
\end{array}\right]=\left[\begin{array}{ccc}
\cos \alpha \cos \beta & \sin \alpha & -\cos \alpha \sin \beta \\
-\sin \alpha \cos \beta & \cos \alpha & \sin \alpha \sin \beta \\
\sin \beta & 0 & \cos \beta
\end{array}\right]\left[\begin{array}{c}
0 \\
R_{y} \\
0
\end{array}\right]=\left[\begin{array}{c}
\sin a \\
\cos a \\
0
\end{array}\right] R_{y}
$$

Lateral force on projectile body is $R_{z}=0.5 \rho S C_{z} V^{2}$. As to aerodynamic axial symmetry projectile, calculation of lateral force is the same as lift force. Projection of lateral force in BCS is

$$
\left[\begin{array}{l}
R_{z x 1} \\
R_{z y 1} \\
R_{z z 1}
\end{array}\right]=L(\beta, \alpha)\left[\begin{array}{c}
0 \\
0 \\
R_{z}
\end{array}\right]=\left[\begin{array}{ccc}
\cos \alpha \cos \beta & \sin \alpha & -\cos \alpha \sin \beta \\
-\sin \alpha \cos \beta & \cos \alpha & \sin \alpha \sin \beta \\
\sin \beta & 0 & \cos \beta
\end{array}\right]\left[\begin{array}{c}
0 \\
0 \\
R_{z}
\end{array}\right]=\left[\begin{array}{c}
-\cos a \sin \beta \\
\sin a \sin \beta \\
\cos \beta
\end{array}\right] R_{z}
$$

Projection of gravity force $\vec{G}_{T}$ in BCS is

$$
\left[\begin{array}{c}
G_{x 1} \\
G_{y 1} \\
G_{z 1}
\end{array}\right]=-m g\left[\begin{array}{c}
\sin \varphi \\
\cos \varphi \cos \gamma \\
-\cos \varphi \sin \gamma
\end{array}\right]
$$

Stabilizing moment $\vec{M}_{z}$ is caused by aerodynamic force to projectile mass center, whose expression is

$$
\vec{M}_{z}=\frac{\rho s l}{2} v_{r}^{2} m_{z}^{\prime}\left(\vec{x}_{3} \times \vec{x}_{1}\right) \frac{\delta_{r}}{\sin \delta_{r}}
$$

And, $m_{z}^{\prime}=m_{z_{a}}+m_{z_{a^{3}}} \delta_{r}^{2}, \quad \vec{x}_{3} \times \vec{x}_{1}=\left[\begin{array}{ccc}i & j & k \\ \cos a \cos \beta & -\sin a \cos \beta & \sin \beta \\ 1 & 0 & 0\end{array}\right]=\left[\begin{array}{c}0 \\ \sin \beta \\ \sin a \cos \beta\end{array}\right]$.

Projection of stabilizing moment in BCS $O x_{1} y_{1} z_{1}$ is

$$
\left[\begin{array}{c}
M_{x 1} \\
M_{y 1} \\
M_{z 1}
\end{array}\right]=\frac{\rho s l}{2} v_{r}^{2} m_{z}^{\prime} \frac{\delta_{r}}{\sin \delta_{r}}\left[\begin{array}{c}
0 \\
\sin \beta \\
\sin a \sin \beta
\end{array}\right]
$$

Where, $\rho$ is air density, $\mathrm{kg} / \mathrm{m}^{3} ; s$ is maximum cross section area, $\mathrm{m}^{2} ; \delta_{r}$ is included angel between projectile axis vector and velocity vector, $\delta_{r}=\cos ^{-1}(\cos a \cos \beta) ; m_{z}^{\prime}$ is derivative of overturning moment coefficient; $m_{z_{a}}$ is one degree term coefficient of overturning moment; $m_{z_{a^{3}}}$ is three degree term coefficient of overturning moment.

Projection of projectile rotational angular velocity and damping moment in BCS are

$$
\left[\begin{array}{c}
\omega_{x 1} \\
\omega_{y 1} \\
\omega_{z 1}
\end{array}\right]=\left[\begin{array}{c}
\dot{\psi} \sin \varphi+\dot{\gamma} \\
\dot{\psi} \cos \varphi \cos \gamma+\dot{\varphi} \sin \gamma \\
-\dot{\psi} \cos \varphi \sin \gamma+\dot{\varphi} \cos \gamma
\end{array}\right],\left[\begin{array}{c}
M_{x z 1} \\
M_{z y 1} \\
M_{z z 1}
\end{array}\right]=\left[\begin{array}{c}
-\frac{\rho v_{r} s}{4} l d m_{x z}^{\prime}(\dot{\psi} \sin \varphi+\dot{\gamma}) \\
-\frac{\rho v_{r} s l}{4} d m_{z z}^{\prime}(\dot{\psi} \cos \varphi \cos \gamma+\dot{\varphi} \sin \gamma) \\
-\frac{\rho v_{r} s l}{4} d m_{z z}^{\prime}(-\dot{\psi} \cos \varphi \sin \gamma+\dot{\varphi} \cos \gamma)
\end{array}\right]
$$

Where, $M_{x z 1}$ is component of pole damping moment along negative $O_{x 1}$ direction of BCS; $M_{z y 1}$ is component of equatorial damping moment along negative $O_{y 1}$ direction of BCS; $M_{z z 1}$ is component of equatorial damping moment along negative $O_{z 1}$ direction of BCS. $m_{z z}^{\prime}$ is derivative of equatorial damping moment coefficient; $m_{x z}^{\prime}$ is derivative of pole damping moment coefficient; $d$ is diameter of projectile, $\mathrm{m} ; l$ is length of projectile, $\mathrm{m}$. 


\section{Simulation and Results Analysis}

Considering meteorological conditions as air temperature, air pressure, wind speed, wind direction, etc. different models M1, M2 and M3 are used to simulate the uncontrolled flight exterior trajectory section of some bomb separately, where, M1 uses BCS-based modeling method [8]; M2 and M3 use ACS-based modeling method, whereas, kinetic models of mass center for M2 and M3 are established on ECS and BCS respectively. Fig.1 and Fig.2 show lateral displacements and altitude results of exterior trajectory simulation respectively, where $x$ axis is flight time (s); $y$ axes are lateral displacement (m) and altitude displacement (m); L1, L2 and L3 correspond to M1, M2, and M3 respectively.

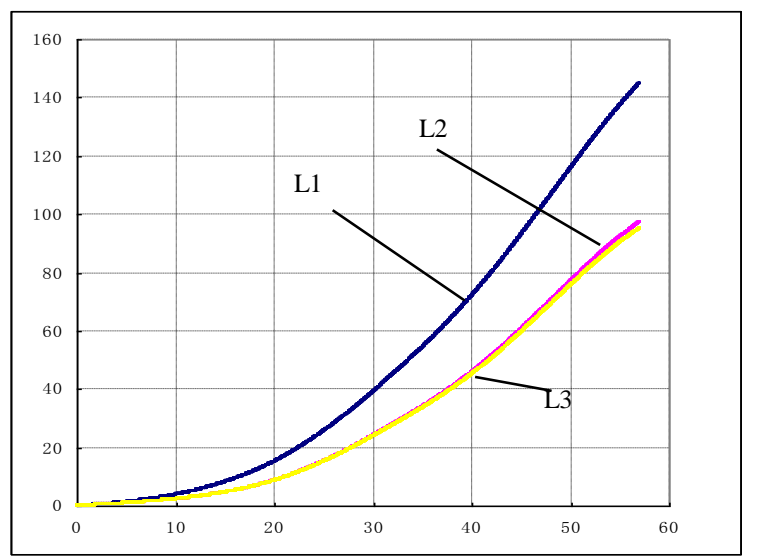

Fig.1. Lateral displacement results of exterior trajectory simulation with 3 models

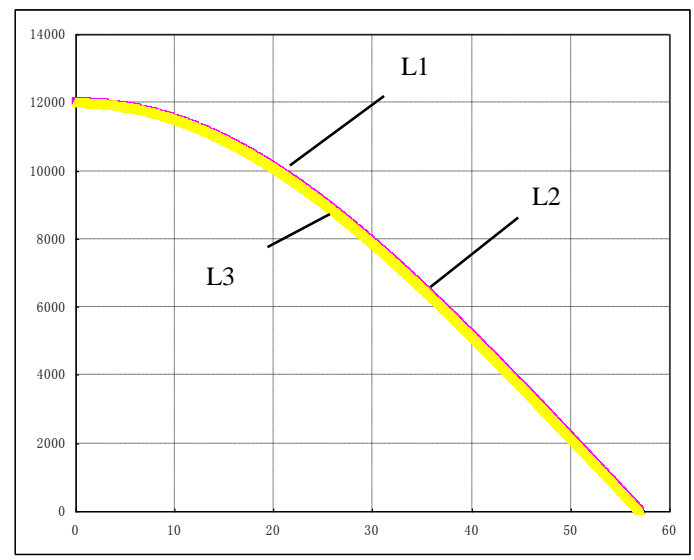

Fig.2. Altitude results of exterior trajectory simulation with 3 models

Table 1 and Table 2 show calculated drop points with 3 models in actual and standard meteorological conditions.

Table 1. Calculated drop points of projectile in actual meteorological condition

\begin{tabular}{|c|c|c|c|}
\hline Model & $\mathrm{X}(\mathrm{m})$ & $\mathrm{Y}(\mathrm{m})$ & $\mathrm{Z}(\mathrm{m})$ \\
\hline M1 & 11182.185 & 0 & 144.844 \\
\hline M2 & 11181.907 & 0 & 95.012 \\
\hline M3 & 11181.951 & 0 & 97.167 \\
\hline
\end{tabular}

Table 2. Calculated drop points of projectile in standard meteorological condition

\begin{tabular}{|c|c|c|c|}
\hline & M1 & M2 & M3 \\
\hline $\mathrm{X}(\mathrm{m})$ & 11108.310 & 11108.4 & 11108.309 \\
\hline $\mathrm{Z}(\mathrm{m})$ & 0 & 0 & 0 \\
\hline
\end{tabular}

It can be seen from Table 1 that calculation error with different exterior trajectory modeling methods cannot be neglected in same actual meteorological condition, especially for those with different reference frame. Such as calculated result of M1 is distinctly larger than those of M2 and 
M3, whereas ACS-based M2 and M3 take minor differences on both distance and lateral displacement. From Table 2, we can see that lateral displacements of 3 models are zero and distance deviations are also very small in standard meteorological condition with zero wind speed, which shows the rationality of model derivation in this paper.

To sum up, different modeling methods for same simulation object take on different errors. In practical applications, suitable modeling method should be selected in allusion to flight rule and aerodynamic configuration of simulation object to decrease error. In this paper, flight features of winged low speed spinning guided bomb as fin stabilization and low speed rolling are considered to choose ACS-based modeling method. In light of terminal control with different control force and moment on projectile body, BCS-based kinematics model and relevant aerodynamic coefficients computation model are deduced, which can provide some basis for subsequent guidance control system simulation.

\section{Conclusions and Prospective}

In simulation test of guided bomb, modeling method has always been a key influencing factor on simulation precision. This paper focuses on modeling method selection problem of exterior trajectory simulation. Application conditions of different exterior trajectory modeling methods are analyzed. And an ACS-based modeling method for certain winged low speed spinning guided bomb is given out for exterior trajectory simulation. On comparing calculation error of different modeling methods, rational modeling method as an efficient path to decrease error and improve simulation precision is illustrated, which provides foundation to guarantee precision of subsequent guidance control system simulation.

\section{References}

[1] Zhao Xichun, Shi Weike. Simulation and flight test integration technique in missile finalized test and evaluation[J]. Modern Defence Technology, 2012: 40(6): 150-154.

[2] Coad P, Yourdon E. Object-oriented design. Prentiee Hall: Yourdon press,1991.

[3] Boock G. Objected-oriented Analysis and Design with Application.2nd ed. Redwood City: Ben-jamin/ Cummings Publishing Company,1994.

[4] Han Zipeng, etc. Exterior ballistics of projectile and rocke. Beijing: Beijing institute of technology press, 2008.

[5] Song Piji. Exterior ballistics of guns and rockets. Beijing: Weapon industry press, 1993.

[6] Qiang Xingfang, Lin Ruixiong, Zhao Yanan. Missile flight aerodynamics. Beijing: Beijing institute of technology press, 2000.08.

[7] Li Xinguo, Fang Qun. Winged missile flight dynamics. Xi'an: Northwestern polytechnical university press, 2008.

[8] Wu Hongquan, He Chi, Wei Hongqiang, et al. Research and development of embed ballistic trajectory simulation system based on Android portable apparatus [J]. Journal of system simulation, 2013: 25(8): 1741-1745. 